\title{
Rédiger contre son opinion : des étudiants avancés en communication peuvent-il faire abstraction de leurs connaissances du domaine?
}

Denis Alamargot et Céline Beaudet

\section{(2) OpenEdition \\ Journals}

Édition électronique

URL : http://journals.openedition.org/pratiques/1466

DOI : $10.4000 /$ pratiques. 1466

ISSN : 2425-2042

Éditeur

Centre de recherche sur les médiations (CREM)

Édition imprimée

Date de publication : 15 décembre 2009

Pagination : 218-232

Référence électronique

Denis Alamargot et Céline Beaudet, «Rédiger contre son opinion : des étudiants avancés en communication peuvent-il faire abstraction de leurs connaissances du domaine? », Pratiques [En ligne], 143-144 | 2009, mis en ligne le 19 juin 2014, consulté le 14 novembre 2019. URL : http:// journals.openedition.org/pratiques/1466; DOI : 10.4000/pratiques. 1466 


\section{Rédiger contre son opinion : des étudiants avancés en communication peuvent-il faire abstraction de leurs connaissances du domaine?}

\section{Denis Alamargot}

Université de Poitiers - CNRS

\section{Céline Beaudet}

Université de Sherbrooke - Québec

\section{Approche Théorique}

\subsection{La rédaction professionnelle ou l'art de la médiation}

La production d'écrits à buts persuasifs en milieu de travail exige du rédacteur la capacité d'adopter une position énonciative qui reflète/reproduit le carrefour d'intérêts au centre duquel il opère. Il ne s'agit donc pas de transmettre par le texte ses propres connaissances d'un domaine, comme l'on serait amené à le faire dans le cas de descriptions, de prescriptions ou d'explications mais de justifier une position sur demande, dans un contexte sociodiscursif préétabli. La question est de savoir comment un rédacteur professionnel, et plus encore un apprenti rédacteur, peut faire preuve de désengagement apparent dans la médiation textuelle d'un savoir fortement orienté et qui, de surcroît, peut être conflictuel avec ses propres valeurs ou opinions.

Le passage entre l'université et le marché du travail s'accompagne souvent d'une découverte importante : les milieux de travail sont hiérarchisés, les enjeux des actions de communication sont économiques, idéologiques, sociaux, politiques. L'action langagière écrite à laquelle le rédacteur professionnel participe contribue à l'atteinte d'objectifs organisationnels particuliers. Dans le cas d'écrits argumentatifs, cela suppose 1'expression d'opinions construites, l'adoption de points de vue concertés, une certaine « mise en scène » de la réalité (Danblon, 2005), qui traduisent la culture ambiante. Le scripteur ou rédacteur (sujet d'énonciation) et l'auteur (sujet réel) inscrits dans une chaîne de commandement polyphonique ne sont pas nécessairement de la même opinion (Plane, 2006). En soi, cette affirmation n'a rien de particulièrement troublante ni étonnante : le savoir-être professionnel comporte une part de séparation de l'être privé et de l'être public, et dans 
le domaine de la communication, la défense d'opinions doit être considérée comme partie intrinsèque de la sphère professionnelle. On n'attend pas du rédacteur professionnel qu'il "épouse corps et âme » les causes sur lesquelles il disserte : il suffit qu'il produise des discours convaincants ( $c f$. Alamargot, Terrier et Cellier, 2007 pour une synthèse).

Pour prendre des exemples dans l'actualité québécoise, il est difficile, à la lecture des rapports annuels d'entreprises d'État comme Loto-Québec et Hydro-Québec ou encore d'une grande institution comme le Centre Hospitalier Universitaire de Montréal (CHUM) de déterminer si le ou les rédacteurs sont contre la nationalisation des jeux de hasard, s'ils ont une quelconque opinion sur la construction de grands barrages hydroélectriques et leur effet sur l'environnement ou sur la privatisation de certains soins de santé jusqu'ici couverts par le système public d'assurance-maladie. Les rapports annuels des institutions et des entreprises, quelle que soit la situation dans laquelle elles se trouvent, traduisent le consensus auquel sont arrivés leurs conseils d'administration et leurs comités de direction et reflètent leurs intérêts et objectifs. C'est une loi du genre. S'il advient que le rédacteur entre en conflit de valeurs avec son employeur, il peut démissionner ou il peut, pour des motifs qu'il appartient à chacun de déterminer, chercher à aménager un espace de conciliation entre son opinion et celle qu'il doit défendre (Donahue, 2005).

Cette distanciation entre sa représentation personnelle (connaissances, croyances, jugements) d'un domaine de connaissances et la schématisation du savoir propre au texte qu'il a à produire entraine le rédacteur dans des procédés d'écriture qui peuvent affecter le texte en surface et en profondeur. La situation exige l'édification d'un plan de rédaction complexe et une représentation de la tâche où l'objectif de conciliation des points de vue (celui de l'auteur et du rédacteur) et des domaines de connaissances l'emporte sur la volonté de confrontation. Les affirmations de Flower, Schriver, Carey, Haas et Hayes (1989) sont intéressantes à ce sujet : «Parfois les conflits sont traités comme des problèmes locaux au sein des textes, dont la résolution suppose l'élimination de l'une des parties conflictuelles. En d'autres circonstances, les conflits deviennent des occasions de construire des plans ou des textes plus intégrés ou élaborés. Toutefois, il semble que les confits ne deviennent une opportunité que si les rédacteurs considèrent leurs buts comme négociables et s'ils adoptent des stratégies de résolution qui génèrent des plans nouveaux ou approfondis lorsque nécessaire » [notre traduction).]

En somme, ni tout à fait rédacteur obéissant à son mandat, ni tout à fait auteur au sens de sujet autonome et sans contraintes, le scripteur écrivant un texte dont les idées entrent en conflit avec ses propres convictions devient alors un être hybride, puisant à même les deux rôles pour se tirer d'affaire. Toutefois, cette habileté exige de lui une grande maîtrise des connaissances et des univers de valeurs propres à chaque communauté discursive et une motivation à « marcher sur la pointe des pieds ».

\subsection{La difficulté de négocier}

Dans une situation de conflit de valeurs, le rédacteur expérimente l'appropriation d'un discours basé sur des faits, connaissances et opinions qui ne correspondent pas à sa représentation habituelle de la réalité. C'est une situation difficile. Conséquemment, il ne faut pas s'étonner qu'il ait été démontré à différentes reprises que soutenir une thèse contraire à ses convictions entraîne une diminution du nombre d'idées dans un texte. Énoncer une position, l'assortir de preuves et formuler la loi de passage qui lie ces preuves à la conclusion sont les étapes les plus cou- 
rantes de l'argumentation ordinaire, tandis que la réfutation de la thèse adverse et des objections anticipées est laissée de côté (Stein \& Miller, 1993a). Cet état de fait est déploré par les magistrats, toujours selon Stein et Miller (1993a), qui reçoivent des avocats des parties en litige des résumés ne comportant aucune piste de réconciliation du conflit. Il s'ensuit que l'habitude est prise, dès le jeune âge, de ne pas réfléchir aux arguments et éléments de contenu à l'appui d'une position contraire à la sienne. Golder (1996) a démontré que l'aptitude à se justifier précédait le développement de l'aptitude à négocier une position chez les enfants, la négociation n'apparaissant possible que vers l'âge de 14 ans, dans les travaux dirigés des élèves. Faisant référence à une enquête menée par Stein et Miller (1993b) auprès d'un groupe d'enfants fréquentant l'école primaire et d'adultes pour vérifier s'ils possédaient autant de connaissances sur la manière d'élaborer des preuves pour soutenir leur thèse que pour réfuter les attaques de l'adversaire, Stein, Bernas, Calicchia et Wright (1996) rapportent qu'il est apparu que les deux groupes avaient beaucoup de difficulté à produire des arguments qui affaiblissaient leur propre position. Autrement dit, ils n'arrivaient pas à changer de position et raisonner dans un domaine de connaissance autre, lors la confrontation argumentative. Il faut en conclure que l'effort cognitif exigé par la négociation d'une position demeure plus important, même à l'âge adulte, que l'effort de se justifier.

\subsection{Vers l'expérimentation}

Nous nous sommes posé deux questions en regard d'un corpus de rédactions étudiantes que nous avons analysé : (i) les effets de l'opinion sont-ils constatés chez des étudiants avancés, ayant une formation à l'écriture professionnelle et qui donc ont pu apprendre à gérer les conflits ; (ii) si ces effets existent chez les étudiants avancés, peut-on en décrire plus précisément les conséquences sur la temporalité rédactionnelle (fluences d'écriture), certaines caractéristiques linguistiques du texte (connecteurs, marqueurs) et les procédés argumentatifs utilisés pour médiatiser le contenu?

Dans le cas qui nous occupe ici, le discours exigé des étudiants est de type argumentatif; l'enjeu pour les étudiants est, d'une part, de gérer la source énonciative (Kerbrat-Orecchioni, 1980 et 1997) qu'ils incarnent, et, d'autre part, d'apprendre à distinguer entre leurs propres connaissances du sujet (souvent approximatives), leurs convictions et l'argumentation à développer selon une situation de communication. Il doivent ainsi préférer la construction d'un discours axé sur le développement d'idées liées à un contexte socio-discursif donné plutôt que de recourir aux lieux communs ou idées reçues qu'ils entretiennent sur le sujet et qui sont à la base d'une justification non dialogale, plus proche des techniques discursives de propagande que de 1'argumentation (Angenot, 2008 ; Garric et Léglise 2005).

Dans le but de vérifier si le fait de rédiger à l'encontre de son opinion laisse des traces temporelles et textuelles, nous proposons de comparer, dans les deux versions d'un même auteur, les fluences de production et la présence de certains indices d'énonciation, soit : les connecteurs à valeur argumentative, les adjectifs et noms subjectifs (marqueurs emphatiques), les adverbes de jugement, les pronoms personnels, les temps verbaux et les verbes impersonnels. Afin d'affiner la description des plans et procédés argumentatif adoptés, notre analyse portera, dans un deuxième temps, sur les doubles versions de 10 auteurs sélectionnés en cherchant à comprendre comment ils réussissent à transformer le contenu des sources documentaires pour constituer le contenu de leur propre texte, en fonction de leur opinion propre. 


\section{Expérimentation}

\subsection{Participants}

17 étudiants en communication, des premier et deuxième cycles universitaires, ont participé à cette expérience, âgés de 28,59 ans $\left(\mathrm{DS}^{(1)}=9,29\right)$, insérés dans 1e cursus universitaire. Leur formation avancée en rédaction les classe dans un groupe dont on peut attendre qu'il ait la capacité d'utiliser les textes sources comme base pour transformer des connaissances et les adapter à une nouvelle situation de communication

\subsection{Tâches expérimentales}

\subsubsection{Lecture de sources documentaires}

Les participants étaient invités à lire deux sources documentaires contradictoires traitant de l'économie et l'environnement : " Gaz à effet de serre : le grand responsable, c'est l'homme » (528 mots) ; «Changements climatiques : la mise au point de nouvelles technologies » (551 mots). L'objectif de cette lecture était de leur permettre d'intégrer deux domaines de connaissance ainsi que d'activer leurs connaissances préalables sur ces thèmes.

\subsubsection{Questionnaire d'opinion}

Un questionnaire d'opinion a été fourni aux participants. Il s'agissait de noter de 1 à 6 («Pas du tout d'accord» à « Tout à fait d'accord») son adéquation avec chacune des 10 propositions présentées dans le questionnaire. La note sur 60 était d'autant plus élevée que les participants partageaient le point de vue du texte écologique.

\subsubsection{Rédaction de deux textes contradictoires}

Les participants étaient invités à rédiger successivement deux textes en adoptant le rôle d'un rédacteur professionnel travaillant soit pour l'association Amis de la Terre (défense du point de vue écologique), soit pour la société BioPétrole (défense du point de vue industriel). Le temps de rédaction était libre mais la longueur de la rédaction imposée (2 pages manuscrites). La consultation des sources documentaires n'était pas autorisée et l'intégration d'idées personnelles encouragée. Les modifications et révisions du texte étaient autorisées. Les participants étaient incités à rédiger une version aboutie dans la mesure où il était indiqué que les deux pages rédigées seront ultérieurement dactylographiées par un secrétaire.

\subsection{Procédure}

Après la passation de la consigne générale, les participants étaient invités à lire les deux sources en vue de la réponse à un questionnaire d'opinion.

A l'issue du questionnaire, les participants étaient invités à rédiger les deux textes sans possibilité de consulter à nouveau les sources. Ils devaient donc rédiger de mémoire, en récupérant les informations des sources et leurs connaissances propres.

Au début et à la fin de la rédaction de chaque texte, les participants notaient l'heure dans un espace réservé. L'ordre de lecture des sources documentaires et de rédaction des deux textes a été systématiquement contrebalancé.

(1) DS : déviation standard - Ecart-type de la distribution. 


\subsection{Variables mesurées}

\subsubsection{Analyse des textes produits}

Les textes produits ont été analysés à travers leurs caractéristiques linguistiques et temporelles.

\section{Description linguistique :}

— nombre de mots du texte : un mot est défini strictement comme une suite de lettres entre deux espaces. La ponctuation n'est pas considérée dans le comptage.

— nombre de paragraphes : un paragraphe est défini par la présence d'un alinéa.

— nombre d'unités d'idées : une unité d'idée est une unité minimale de sens, qui apporte une information supplémentaire ou complémentaire.

- nombre de mots par paragraphes.

— nombre de mots par unités d'idées.

- Verbes :

- fréquence des verbes (en fonction du nombre de mots),

- répartition en fonction du temps (passé, présent, futur),

- répartition en fonction du sujet (personnel, impersonnel, modal) : un verbe est considéré comme personnel lorsque son sujet a un contenu sémantique (exemple : le renard mange un fromage), comme impersonnel lorsque son sujet, le pronom il, n'est qu'une position syntaxique vide de sens. (Il pleut) ; parmi les verbes impersonnels se trouvent les verbes modaux, qui expriment un point de vue sur le verbe prédicatif ou la proposition qui les suivent (exem-

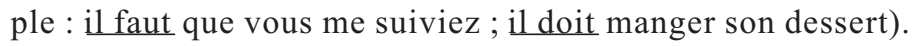

— Marqueurs d'emphase et d'intensité :

- fréquence des marqueurs (en fonction du nombre de mots).

- répartition catégorielle (adjectifs et noms subjectifs, verbes emphatiques (personnels, impersonnels, modaux, adverbes et adjectifs d'intensité). Les marqueurs emphatiques correspondent au vocabulaire subjectif qui ne traduit pas des caractéristiques objectivables de la réalité mais le point de vue de celui qui observe la réalité. Exemples de vocabulaire emphatique : adjectifs subjectifs : odieux, catégorique, rigide, alarmants. Noms (de qualité) : normalité, barbares, pourritures, technologie verte. Verbes personnels : détrompez-vous, ils balaient du revers de la main, nous savons que, enfourner la planète. Verbes modaux : il serait illusoire de croire, il ne fait aucun doute que, il faut faire ; verbes impersonnels : il en revient à chacun de nous. Adverbes d'intensité : extrêmement, doublement, nécessairement. Adjectifs d'intensité : quantité atmosphérique, denrée rare, pollution extrême.

- Connecteurs :

- fréquence des connecteurs (en fonction du nombre de mots).

- répartition catégorielle et exemples : addition : de plus, aussi, de surcrô̂t ; d'opposition : Plutôt que, par opposition, mais ; cause : parce que, en raison de, c'est pourquoi, car; conséquence : par conséquent, en effet, donc ; concession : certes; but : en vue de, vers.

- Pronominalisation :

- Fréquence des pronoms (en fonction du nombre de mots). 
Mesures temporelles:

— durée de la tâche (en sec).

- débits (sec par unités) :

- temps par mots,

- temps par paragraphe,

- temps par idées.

\section{Résultats}

\subsection{Questionnaire d'opinion}

La moyenne du jugement est égale à $46.65(\mathrm{DS}=5,21)$ avec un score minimum de 37 et maximum de 54 . La médiane est égale à 48 , le mode à 47 . Il ressort que tous les participants sont favorables à une position écologiste (score minimum supérieur à 30) même si une variation au sein de groupe peut être décrite.

\subsection{Analyse quantitative des textes produits}

Des analyses statistiques ont été conduites pour apprécier les effets de 1'opinion sur les caractéristiques linguistiques et temporelles des textes. Au regard des résultats au questionnaire, le facteur «Opinion » a été constitué de la façon suivante : Rédiger pour Les amis de la Terre est considéré comme rédiger selon son opinion, en pouvant recourir à son propre domaine de connaissance; rédiger pour BioPétrole est considéré comme rédiger contre son opinion. Des analyses de $t$ de Student pour échantillons appariés ont été conduites pour apprécier l'effet du facteur «Opinion» sur les différentes mesures. Pour rendre compte de la variation des opinions au sein du groupe de participants, des analyses de corrélations entre l'opinion au questionnaire et les différentes mesures ont été menées. Enfin, l'effet du contrebalancement de l'ordre de présentation des sources documentaires et de rédaction des textes a été évalué par le biais de t de Student. Il n'existe pas d'effet significatif, sur les différentes variables mesurées, de l'ordre de lecture de la source documentaire, ni de l'ordre de rédaction des textes.

\subsubsection{Sur le plan linguistique}

Tableau 1 - Moyenne et (DS) des différentes variables linguistiques selon l'opinion ( $N=17$ participants).

\begin{tabular}{|l|r|r|r|r|}
\hline \multirow{2}{*}{} & \multicolumn{4}{|c|}{ Opinion } \\
\cline { 2 - 6 } & \multicolumn{2}{|c|}{$\begin{array}{c}\text { Pour } \\
\text { (Les Amis de la Terre) }\end{array}$} & \multicolumn{2}{c|}{$\begin{array}{c}\text { Contre } \\
\text { (Bio Pétrole) }\end{array}$} \\
\hline Nombre de mots & 292.82 & $(45.53)$ & 258.53 & $(43.04)$ \\
\hline Nombre de paragraphes & 3.65 & $(1.27)$ & 3.76 & $(1.60)$ \\
\hline Nombre d'idées & 27.12 & $(5.01)$ & 23.82 & $(4.11)$ \\
\hline Nombre de mots par paragraphes & 94.51 & $(50.62)$ & 93.71 & $(78.94)$ \\
\hline Nombre de mots par idées & 10.98 & $(1.76)$ & 10.94 & $(1.33)$ \\
\hline Verbes au passé & 13.85 & $(8.98)$ & 12.33 & $(6.96)$ \\
\hline Verbes au présent & 80.02 & $(10.44)$ & 83.28 & $(7.78)$ \\
\hline Verbes au futur & 6.12 & $(7.66)$ & 4.395 & $(3.58)$ \\
\hline Verbes au total & .106 & $(.023)$ & .117 & $(.019)$ \\
\hline Verbes impersonnels & 11.55 & $(6.96)$ & 12.50 & $(8.60)$ \\
\hline Verbes personnels & 77.59 & $(8.15)$ & 78.36 & $(8.38)$ \\
\hline Verbes modaux & 10.86 & $(6.28)$ & 9.14 & $(5.08)$ \\
\hline
\end{tabular}




\begin{tabular}{|l|r|r|r|r|}
\hline Marqueurs emphatiques (Adjectifs) & 18.92 & $(22.32)$ & 16.41 & $(23.47)$ \\
\hline Marqueurs emphatiques (Noms) & 10.50 & $(18.35)$ & 12.56 & $(21.52)$ \\
\hline Marqueurs emphatiques (Verbes) & 13.21 & $(22.46)$ & 0 & 0 \\
\hline Marqueurs d'intensité (Adjectifs) & 57.37 & $(26.30)$ & 71.03 & $(32.41)$ \\
\hline Marqueurs emphatiques/intensité au total & .018 & $(.010)$ & .008 & $(.007)$ \\
\hline Connecteurs d'additivité & 59.71 & $(20.85)$ & 45.16 & $(18.75)$ \\
\hline Connecteur d'opposition & 7.62 & $(13.24)$ & 12.05 & $(13.70)$ \\
\hline Connecteurs de causalité & 14.28 & $(11.68)$ & 13.14 & $(17.25)$ \\
\hline Connecteurs de conséquence & 5.94 & $(9.79)$ & 6.21 & $(6.93)$ \\
\hline Connecteurs de concession & 4.68 & $(11.57)$ & 11.22 & $(12.88)$ \\
\hline Connecteur de but & 7.78 & $(12.93)$ & 12.21 & $(14.86)$ \\
\hline Connecteurs au total & .022 & $(.007)$ & .026 & $(.006)$ \\
\hline Nombre de pronoms & .014 & $(.010)$ & .016 & $(.008)$ \\
\hline
\end{tabular}

Les analyses statistiques montrent que les participants, lorsqu'ils rédigent un texte en accord avec leur opinion, produisent un plus grand nombre : de mots (Moy «Pour $»=292.82, \mathrm{DS}=45.53 ;$ Moy $«$ Contre $»=258.52, \mathrm{DS}=43.02 ; \mathrm{t}=4.07, \mathrm{df}$ $=16, p<.001)$; d'idées (Moy «Pour $»=27.12, \mathrm{DS}=5.00 ;$ Moy $«$ Contre $»=23.82$, $\mathrm{DS}=4.11 ; \mathrm{t}=2.96, \mathrm{df}=16, p<.01)$ et de marqueurs d'emphase ${ }^{(2)}$ (Moy « Pour» $=.018, \mathrm{DS}=.01 ;$ Moy $«$ Contre $»=.008, \mathrm{DS}=007, \mathrm{t}=3.51, \mathrm{df}=16, p<.003)$. En revanche, ils produisent significativement moins de connecteurs (Moy "Pour» $=.022, \mathrm{DS}=.07 ;$ Moy $«$ Contre $»=.026, \mathrm{DS}=006, \mathrm{t}=3.51, \mathrm{df}=16, p<.04)$ et, en terme de tendance, relativement moins de connecteurs d'additivité (Moy « Pour» $=59,71 \%, \mathrm{DS}=20,85 \% ;$ Moy $«$ Contre $»=45,16 \%, \mathrm{DS}=18.55 \%, \mathrm{t}=2.10, \mathrm{df}=16$, $p<.051)$. Aucun autre effet significatif ou tendanciel de l'opinion n'a pu être relevé.

\subsubsection{Sur le plan temporel}

Tableau 2 - Moyenne et (DS) des différentes variables temporelles selon l'opinion $(N=17$ participants $)$.

\begin{tabular}{|l|r|r|r|r|}
\hline \multirow{2}{*}{} & \multicolumn{4}{|c|}{ Opinion } \\
\cline { 2 - 5 } & \multicolumn{2}{|c|}{$\begin{array}{c}\text { Pour } \\
\text { (Amis de la Terre) }\end{array}$} & \multicolumn{2}{c|}{$\begin{array}{c}\text { Contre } \\
\text { (BioPétrole) }\end{array}$} \\
\hline Durée de la tâche (en sec) & 1958.82 & $(736.66)$ & 2075,29 & $(1355)$ \\
\hline Débit mot (temps par mots en sec) & 6.88 & $(2.82)$ & 7.94 & $(4.52)$ \\
\hline Débit paragraphe (temps par paragraphes en sec) & 598.71 & $(269.35)$ & 760.03 & $(791.69)$ \\
\hline Débit idées (temps par idées en sec) & 74.85 & $(31.02)$ & 87.04 & $(54.34)$ \\
\hline
\end{tabular}

Aucun effet significatif de l'opinion n'a pu être relevé concernant les variables temporelles.

\subsubsection{Corrélations entre le score d'opinion et l'ensemble des variables linguistiques et temporelles}

L'analyse des corrélations (coefficient de Bravais-Pearson) entre le score d'opinion au questionnaire et l'ensemble des variables (linguistiques et temporelles)

(2) Il est à noter que seulement 13 participants sur 17 produisent au moins 1 marqueur d'emphase dans le cas d'un désaccord avec le thème du texte alors que tous les sujets en produisent dans le cas d'un accord. 
montre qu'une augmentation de l'opinion favorable à la position écologiste est associée, en termes de tendance, (i) à une diminution du nombre d'idées dans les textes défendant la position opposée (Rédiger pour «BioPétrole») $(\mathrm{r}=-.55 ; p=.054)$ ainsi qu'à (ii) une diminution du nombre de pronoms dans les textes défendant la position associée (Rédiger pour Amis de la Terre) $(\mathrm{r}=-.41 ; p<.10)$.

\subsection{Analyse qualitative des procédés d'argumentation utilisés}

L'analyse quantitative montre que l'effet de l'opinion ne joue ici que sur les caractéristiques linguistiques du texte. Rédiger contre son opinion conduit à produire un texte plus court, comportant moins d'idées et de marqueurs emphatiques mais un plus grand nombre de connecteurs se composant, en termes de tendance, d'un pourcentage plus faible d'additifs. La question est d'identifier, au niveau du contenu textuel et des procédés argumentatifs, la conséquence des modifications sur le plan de l'utilisation des marqueurs et connecteurs.

Pour ce faire, nous avons conduit une analyse qualitative sur un nombre restreint de textes. Dix sujets parmi les 17 initiaux ont été retenus en raison du fait qu'ils ont produit chacun deux rédactions de longueur égale, suffisamment longues pour présumer que le contenu y est développée de manière à soutenir une analyse significative. De plus, la moitié d'entre eux était inscrite au baccalauréat en Communication, rédaction et multimédia, et l'autre en maîtrise en Communication et langages. L'analyse a consisté à repérer dans chacun des 20 textes produits, phrase par phrase, les arguments et unités d'idées auxquels les scripteurs ont eu recours pour soutenir leurs thèses. Les arguments et unités d'idées ont été classés en utilisant la nomenclature proposée par Angenot (2008, 1982), Beaudet (2005), Perelman et Olbrechts-Tyteca (1988), Plantin (1996) et Robrieux (2000).

L'objectif de l'analyse a été d'évaluer dans quelle mesure les sujets ont pu reprendre ou pas, transformer ou pas, les connaissances fournies par les deux textes source auxquels ils avaient accès, en fonction de leur opinion. Les textes source incitent le rédacteur à vérifier les contenus factuels, à les reprendre de manière rigoureuse, ou non. Ils lui permettent aussi, s'il en a la capacité, de profiter des contenus accessibles par la lecture pour en développer de nouveaux, d'associer des connaissances personnelles à ce qu'il a lu. Le premier texte source comprenait des arguments favorables à une vision environnementaliste ; le deuxième texte argumentait en faveur de l'expansion économique et des investissements technologiques comme solution au développement durable.

\section{- Rédiger selon son opinion : Amis de la Terre}

L'appel à l'action, premier argument par ordre d'importance dans les rédactions Amis de la Terre, n'existe pas dans le texte source 1. Cet argument est né de l'adaptation à la situation de communication : écrire pour une association de défense de l'environnement. Les participants ont interprété leur mandat comme étant pragmatique. Cet argument établit aussi un lien dialogal entre l'énonciateur et ses destinataires.

Les preuves factuelles, d'autorité et par l'avenir sont reprises du texte source 1, à la différence près qu'elles sont dénuées d'explications, alors que dans le texte source, les preuves et sources d'autorité sont élaborées et forment ensemble une théorie. Dans les rédactions des participants, les preuves sont additionnées dans le but de produire un effet rhétorique, de pousser à l'action en agitant la peur de la catastrophe imminente; le premier texte source a une visée explicative plutôt que persuasive. Il s'adresse à « l'intelligence plutôt qu'aux émotions ». 
Dans les rédactions des participants, les exemples illustrent l'appel à l'action. Dans le texte source 1, les exemples ont une valeur explicative en regard des données qui fondent la théorie du réchauffement climatique.

Il était suggéré d'énoncer des arguments de réfutation dans la consigne de rédaction. Les participants se sont réfugiés dans des arguments tels la contradiction, ad personam et l'incompatibilité, qui se nourrissent d'un amalgame d'informations tirées du texte source 2 (mettant en scène l'ancien président Bush et le premier ministre canadien Harper) et de leurs propres connaissances. Ces arguments confèrent un ton polémique aux rédactions et, en guise de réfutation de l'opinion adverse, les rédacteurs ont privilégié la théorie de la conspiration contre le bon sens, l'évidence qu'il n'y a d'autre approche valable que l'approche préconisée par les Amis de la Terre.

On constate donc que les connaissances puisées dans les textes sources ont été transformées, plutôt que simplement rapportées, du fait que les participants ont donné à leur rédaction un but adapté à la situation de communication où ils étaient plongés. Ils ont sélectionné les informations qui concordaient avec ce but et ordonné les arguments en conséquence. En surface, le texte source a orienté fortement le contenu des rédactions, mais le circuit argumentatif a été transformé en profondeur. Les participants ont fait intervenir leurs propres idées reçues sur le sujet et ont raccordé leurs arguments en utilisant comme fil conducteur la peur de la catastrophe imminente. Les données factuelles s'insèrent à l'intérieur d'un discours alarmiste d'où les explications sont absentes. C'est la rhétorique lexicale, omniprésente comme l'a démontré l'analyse statistique, qui l'emporte comme stratégie persuasive. Les arguments rationnels ne le sont qu'en apparence, le recours à la rhétorique langagière servant d'instrument principal pour créer l'idée de peur et de catastrophe.

\section{- Rédiger contre son opinion : BioPétrole}

Si l'on examine l'effet des textes source sur la rédaction BioPétrole, la thèse est empruntée au texte source 2 de même que l'argument de la fin et des moyens. L'incompatibilité apparente des thèses adverses (sauver la planète et soutenir l'expansion de l'économie de consommation), la preuve factuelle et la preuve par l'avenir sont également prises dans le texte source 2. Par contre, l'importance accordée à l'éthos de l'énonciateur (déplacement du logos vers l'ethos) et l'argument d'inclusion (malgré les apparences, BioPétrole adhère aux valeurs environnementalistes) porteur de négociation plutôt que de confrontation) sont des stratégies discursives adaptatives et ne sont pas dans le texte source 2. L'effort de négociation est ensuite contredit, en conclusion, par le caractère autoritaire de l'argument de vérité, emprunté au texte source 2.

Au total, on s'aperçoit que le circuit des arguments du texte source 2 est perturbé par la volonté des participants de réconcilier les points de vue adverses. Les emprunts au texte 2 sont plus marqués que dans la rédaction Amis de la Terre, mais les ajouts et adaptations apportés par les rédacteurs témoignent du fait qu'ils sont tiraillés entre deux points de vue. Il s'ensuit que les rédactions manquent de cohérence. Pour ces apprentis rédacteurs, écrire contre leur propre opinion constitue une expérience de dissociation avec leurs connaissances antérieures et croyances personnelles, et les entraîne à structurer des arguments dans deux schémas d'ensemble qui se contredisent et qu'ils n'arrivent pas à unifier, d'où sans doute l'emploi plus important de connecteurs (pour donner l'impression qu'ils savent dans quelle logique ils s'inscrivent) constaté par l'analyse quantitative ( $c f$. plus haut). 


\section{Interprétation discussion}

Finalement, (i) l'analyse quantitative a montré que la différence entre les deux textes (pour et contre) se marque essentiellement au niveau du contenu (nombre d'idées) et de sa structuration linguistique (connecteurs, marqueurs). La défense de sa propre opinion et de son propre domaine de connaissance suscite une élaboration plus emphatique du contenu alors que la défense d'une opinion adverse conduirait à élaborer un texte plus encyclopédique et factuel, basé sur un nombre limité d'idées mais plus fortement articulées par un nombre plus important de connecteurs.

Comme attendu, rédiger contre son opinion limite le nombre d'idées. Cette moindre aisance à contre-argumenter, explicitée par Stein, Bernas, Calicchia et Wright (1996), peut être due à l'effet des connaissances dans le domaine de référence, effet qui a été mis en évidence à différentes reprises dans la littérature. Ainsi, l'expertise référentielle, en suscitant une densité et une intégration des connaissances en mémoire à long terme plus importantes, permet au rédacteur de composer un texte au contenu plus riche (Caccamise, 1987 ; Voss, Vesonder et Spilich, 1980 ; pour une synthèse : Alamargot, Chanquoy et Chuy, 2005). Le fait que les textes pour et contre se distinguent également sur le plan du nombre des connecteurs et de la nature des marqueurs (emphatiques) trouve une explication par l'analyse qualitative, notamment dans le choix de deux modes d'argumentation différents, conduisant à des exploitations différentes des connaissances contenues dans les sources.

Le fait qu'aucun effet ne soit recueilli sur la temporalité de la production est ici plus surprenant. Rédiger à partir d'un domaine familier réduit en général la durée des pauses d'écriture (Alamargot, 1997 ; Caccamise, 1987 ; Dansac et Alamargot, 1999) ainsi que le coût des processus, notamment de planification (Kellogg, 1987). Il semble ici que les deux textes soient produits avec la même temporalité et la même aisance. Il est probable que cette absence de différence soit due à l'expertise avancée des participants. À ce stade de leur cursus, ils ont pu acquérir et procéduraliser des stratégies d'élaboration du contenu du texte (Alamargot \& Fayol, 2009 pour une synthèse) leur permettant de rédiger de façon optimale, sans procéder à de longues périodes de réflexion ou de révision profonde de leur texte $(c f$. Alamargot, Plane, Lambert \& Chesnet, 2009). Il est possible toutefois que les indicateurs temporels retenus ici restent trop généraux. Le recours à 1'enregistrement des pauses et débits d'écriture tout au long de l'activité (via notamment une tablette à digitaliser et le logiciel Eye and Pen : Chesnet \& Alamargot, 2005 ; Alamargot, Chesnet, Dansac \& Ros, 2006) permettrait une analyse des pauses précédant les idées, les paragraphes ou encore les marqueurs emphatiques et les connecteurs, unités linguistiques ici sensibles. Il s'agit là d'une piste à envisager pour spécifier les traitements rédactionnels sensibles à l'opinion.

(ii) L'analyse qualitative de l'argumentation confirme les conclusions de l'analyse quantitative. Rédiger contre son opinion conduit à produire un texte plus court et comportant moins d'idées mais plus fortement connectées. Cela peut sans doute être lié au fait que, contrairement à ce qui était le cas dans les rédactions Les amis de la Terre, les participants n'ont pas pu recourir à leur connaissances encyclopédiques sur le domaine ou encore à leur répertoire d'idées reçues, telles que véhiculées par le discours social ambiant. L'analyse qualitative laisse penser que les participants ont écrit sans planification d'ensemble du problème et de la tâche, s'attachant plutôt à la cohésion qu'à la cohérence (emphase sur les aspects grammaticaux et syntaxiques, sur l'usage de connecteurs). Une des raisons que nous 
pouvons invoquer hypothétiquement serait l'impossibilité dans laquelle se trouvaient les participants d'imaginer un auditoire à leur rédaction BioPétrole. L'expérimentation, réalisée en milieu universitaire, comportait ses limites. En situation réelle de travail, auraient-ils fait l'effort de résoudre ce problème ?

\section{Conclusion - Perspectives}

Cette expérience et les analyses conduites montrent que les étudiants avancés dans un cursus en communication, rédaction et multimédia sont sensibles à leur propre opinion et domaine de connaissance lorsqu'ils rédigent un texte. Cette influence apparait sur le plan du contenu, des connecteurs et marques linguistiques utilisés et des procédés d'argumentation. Leur expertise avancée en techniques rédactionnelles limiterait toutefois l'impact de l'opinion sur la temporalité de la rédaction (fluence de production) en leur permettant de convoquer des procédures de rédaction "ready made, prêtes-à-porter ». La question qui découle et dont il s'agit de discuter ici porte sur l'enseignement de la rédaction professionnelle et notamment la façon de permettre aux étudiants de conscientiser l'influence de leur propre opinion et connaissance dans le cadre de la rédaction professionnelle.

\subsection{L'enseignement de l'écriture située}

Dans les écrits fonctionnels, soutenir un point de vue sur la réalité, n'importe lequel et pas nécessairement le sien, constitue la norme plutôt que l'exception. Ces écrits ont un ancrage social particulier et, dans la grande majorité des cas, ils ont une visée persuasive (Larson, 1994). Dès lors, les cours de rédaction professionnelle doivent avoir pour visée de développer chez les étudiantes et les étudiants un esprit critique par rapport à leurs productions écrites. L'argumentation est située dans un contexte, elle ne peut pas surgir toute faite (même s'il s'agit d'un sujet connu) et se déplacer d'un texte à l'autre ; elle procède d'une phase de planification de la tâche à accomplir qui affecte le choix du domaine de connaissances, des arguments qui en découlent et leur agencement.

Les cours de rédaction professionnelle à l'université devraient avoir pour visée première de développer chez les étudiantes et les étudiants un esprit critique par rapport aux productions communicationnelles et langagières non littéraires, de types fonctionnels, à buts utilitaires. Dans le monde francophone, la tendance est d'axer l'enseignement sur la transmission de règles et de procédures. Pourtant, les textes utilitaires sont des actions langagières qui font partie de la dynamique des organisations : par conséquent, ils doivent être le produit d'une planification approfondie, basée sur la compréhension des enjeux de la communication. C'est ce qui correspond au concept d'écriture située ou «New Literacy Studies », fondé sur la prémisse suivant laquelle " reading, writing and meaning are always situated within social practices within specific Discourses »(Gee, 1996: 189). [La lecture, l'écriture et le sens sont toujours situés dans des pratiques sociales prenant place dans des communautés discursives spécifiques. (Notre traduction)]

\subsection{Apprendre à entrer dans une communauté de discours}

Le défi principal demeure d'enseigner l'écriture pratiquée en situation professionnelle dans un contexte universitaire, où le rédacteur est d'abord étudiant (Beaufort, 2007 ; Dias, Freedman, Medway et Paré 1999; Spilka, 1993). Écrire efficacement des textes fonctionnels à orientation persuasive exige du rédacteur qu'il se distancie de ses propres connaissances et opinions, qu'il analyse la situa- 
tion de communication pour savoir d'abord de qui (le mandant) il se fait l'intermédiaire. Cette relation entre mandant et rédacteur constitue le premier pôle de la communication professionnelle (Beaudet et Clerc, à paraître) et elle orientera de nombreuses décisions au cours du processus d'écriture. Si on considère l'écriture sur le plan du processus qui lui fait prendre forme (Bereiter et Scardamalia, 1987 ; Garcia-Debanc et Fayol, 2002 ; Hayes \& Flower, 1980 ; Hayes, 1996), c'est à l'étape de la planification des contenus que le rédacteur construit un schéma global du texte à écrire. Il s'ensuit un ensemble de choix sur la visée de l'action langagière, de la structure globale du texte qui convient à cette visée, du genre d'écrit approprié, des idées sélectionnées et des idées à rejeter. Comme le disent Olive et Piolat (2003 : 201) : "Les savoirs linguistiques ou thématiques ne sont pas uniquement en jeu. Le contrôle de la qualité d'un texte relève de nombreuses conditions du traitement de l'information textuelle. "

En effet, quel que soit le message à transmettre, le rédacteur véhicule l'image d'un organisme, d'une entreprise, il traduit une idéologie, des valeurs, une vision du monde qui ne sont pas nécessairement les siennes mais qui émanent d'une communauté de discours spécifique qu'il doit apprendre à reproduire s'il fait partie de ce milieu. Cette capacité d'entrer dans une communauté discursive (Maingueneau $2002: 105$; Swales, $1996: 67$ ) est le résultat d'un apprentissage de haut niveau (Beaufort 2007 ; MacKinnon, 1993), d'où l'expérimentation décrite ici et la réflexion qu'elle suscite sur le plan didactique.Cet apprentissage inclut des notions avancées d'analyse linguistique de discours (Amossy, 2000 ; Bronckart, 1996 ; Charaudeau et Maingueneau, 2002 ; Fairclough, 2009 ; Maingueneau, 1991 ; Vignaux, 1988, pour ne nommer que ces quelques auteurs), par lequel l'étudiant apprend à reconnaître dans un discours déjà construit les procédés de langage (liés au lexique) et de discours (liés à la mise en texte) qui permettent d'en orienter le sens pour ensuite reconnaître et reproduire les pratiques discursives du milieu de travail où il s'insèrera. Selon Maingueneau, "les modes d'organisation des hommes et de leurs discours sont indissociables, les doctrines sont inséparables des institutions qui les font émerger et les maintiennent. Cette hypothèse concerne au premier chefles groupes de producteurs de textes, qui ne doivent pas être considérés comme des médiateurs transparents " (Charaudeau et Maingueneau, $2002: 105$ ). Le rédacteur est responsable du texte, dans tous les sens du terme (Beaudet, 2003).

La qualité du texte produit en milieu de travail est très souvent synonyme de conformité avec la culture dominante de l'entreprise ou de l'institution où le texte prend place, comme le soulignent par ailleurs les mêmes auteurs. Cette tendance est d'autant plus lourde que les textes professionnels sont soumis à une chaîne de lecteurs qui examinent le contenu et vérifient son adéquation avec l'esprit des lieux. Écrire contre son opinion et médiatiser un domaine de connaissance qui n'est pas sien n'est qu'une facette invisible du travail du rédacteur professionnel, mais elle requiert la maîtrise du processus de rédaction dans ses aspects les plus subtils. 


\section{Bibliographie}

Alamargot, D. (1997) : Processus de récupération et d'organisation dans l'activité de rédaction de texte. Rôle du développement des connaissances référentielles. Thèse de Doctorat de Psychologie. Université de Poitiers. Janvier 1997.

Alamargot, D., Chanquoy, L. \& Chuy, M. (2005) : «L'élaboration du contenu du texte : de la mémoire à long terme à l'environnement de la tâche », Psychologie française 50/3, pp. 287-304.

Alamargot, D., Chesnet, D., Dansac, C. \& Ros, C. (2006) : « Eye and Pen : a new device to study reading during writing », Behavior Research Methods, Instruments and Computers 38/2, pp. 287-299.

Alamargot, D., Terrier, P. \& Cellier, J.-M. (eds.) (2007) : Studies in Writing. Written Documents in the Workplace. Amsterdam, Elsevier.

AlAmARGOT, D. \& FAYOL, M. (2009) : « Modelling the development of written composition », Beard, R., Myhill, D., Nystrand, M., Riley, J. (eds), Handbook of Writing Development, Sage, United Kingdom, pp. 23-47.

Alamargot, D., Plane, S., LAmbert, E. \& Chesnet, D. (sous presse) : «Using Eye and Pen movements to trace the development of writing expertise : case studies of a seventh, ninth and twelfth grader, graduate Student, and professional writer ", Reading and Writing.

AMOSSY, R. (2000) : L'argumentation dans le discours : discours politique, littérature d'idées, fiction, Paris, Nathan Université.

ANGENOT, M. ([1982], 1995) : La parole pamphlétaire, Paris, Payot.

— (2008) : Dialogue de sourds. Traité de rhétorique antilogique, Paris, Éditions des mille et une nuit.

BEAUDET, C. (2003) : «Le rédacteur et la fabrication du sens d'un texte persuasif », Communication : information, médias, théories, pratiques 22/2, pp. 44-61.

- (2005) : Stratégies d'argumentation et impact social : le cas des textes utilitaires, Québec, Nota bene.

BEAUDET, C. \& CLERC, I. (à paraître) : «L'enseignement de la rédaction professionnelle au Québec : Quels fondements disciplinaires ? Quelle reconnaissance institutionnelle ? », Actes de la Conférence Internationale « De la France au Québec. L'écriture dans tous ses états ». Du 12 au 15 novembre, 2008. Poitiers - France.

BEAUFORT, A. (2007) : College Writing and Beyond. A New Framework for University Writing Instruction, Logan, Utah, Utah State University Press.

- (2008) : "Writing in the professions », Bazerman, C. (ed.), Handbook of Research on Writing. History, Society, School, Individual, Text, New York, Lawrence Erlbaum Associates, pp. 221-236.

Bereiter, C. \& SCARdamalia, M. (1987) : The Psychology of Written Composition, Hillsdale, NJ, Lawrence Erlbaum Associates.

BRONCKART, J.-P. (1996) : Activité langagière, textes et discours. Pour un interactionnisme socio-discursif, Lausanne, Delachaux et Niestlé.

CACCAMise, D. J. (1987) : «Idea generation in writing », Matsuhashi, A. (ed.), Writing in Real Time: Modelling Production Processes, Norwood, Ablex Publishing Corporation, pp. 224-253.

Charaudeau, P. \& Maingueneau, D. (2002) : Dictionnaire d'analyse de discours, Paris, Seuil.

Chesnet, D. \& Alamargot, D. (2005) : «Analyse en temps réel des activités oculaires et grapho-motrices du scripteur. Intérêt du dispositif "Eye and pen" ", L'année psychologique 105/3, pp. 477-520.

DANBLON, E. (2005) : La fonction persuasive. Anthropologie du discours rhétorique: origines et actualité, Paris, Armand Colin. 
DANSAC, C \& AlAmARGOT, D. (1999) : «Accessing referential information during text composition: when and why? », in M. Torrance \& D. Galbraith (Eds). Knowing what to write: Cognitive processes in the generation, selection and development of ideas during text production, Amsterdam, Amsterdam University Press, pp. 79-97.

Dias, P., Freedman, A., Medway, P. \& Paré, A. (eds.) (1999) : Worlds Apart. Acting and Writing in Academic and Workplace Contexts, Mahwah, NJ, Lawrence Erlbaum Associates Publishers,

Donahue, C. (2005) : «Student writing as Negotiation », Kostouli, T. (ed.), Writing in Context: Textual Practices and Learning Processes in Sociocultural Settings, Studies in writing, 15, New York, Springer, pp. 137-163.

FAIRClOUgh, N. (2009, $2^{\text {nd }}$ edition) : Critical Discourse Analysis, London, Longman.

Flower, L., SCHriver, K.A., CAREY, L., HAAS, C. \& HAYES, J.R. (1989) : «Planning in Writing : The Cognition of a Constructive Process », Technical Report no 34, National Center for the Study of Writing.

GARCIA-DEBANC, C. \& F AYOL, M. (2002) «Apports et limites des modèles du processus rédactionnel pour la didactique de la production écrite. Dialogue entre psycholinguistes et didactitiens », Pratiques 115-116, pp. 37-50.

GARRIC, N. \& LÉGLISE, I. (2005) : « Le discours patronal, discours patronal », Banks, D. (coord.), Aspects linguistiques du texte de propagande, Paris, L'Harmattan, pp. 133-146.

GEE, J.P. (2000) : «The new literacy studies : from "socially situated" to the work of the social », Barton, D. Hamilton, M. \& Ivanic, R. (eds), Situated Literacies. Reading and Writing in Context, London and New York, Routledge, pp. 180-196.

GOLDER, C. (1996) : Le développement des discours argumentatifs, Lausanne, Delachaux et Niestlé.

HAYES, J.R. (1996) : « A new framework for understanding cognition and affect in writing », Levy, M. \& Randell, S. (eds.), The Science of Writing. Theories, Methos, Individual Differences, and Applications, Mahwah, NJ, Lawrence Erbaum Associates, pp. 1-28.

HAYES, J.R. \& FLOWER, L.S. (1980) : «Identifying the organization of writing processes », Gregg, L.W. \& Steinberg, E.R. (eds.), Cognitive Processes in Writing, Hillsdale, NJ, Lawrence Erlbaum Associates, pp. 3-30.

KellogG, R. T. (1987) : «Effects of topic knowledge on the allocation of processing time and cognitive effort to writing processes », Memory and Cognition 15/3, pp. 256-266.

Kerbrat-Orecchioni, C. (1980) : L'énonciation, Paris, Armand Colin.

LARSON, R. (1994) : Curricula in College Writing Programs : Much Diversity, Little Assessment. A report to the Ford Foundation on the Project on College Curricula in Composition.

MACKINNON, J. (1993) : «Becoming a rhetor : Developing writing ability in a mature, writing-intensive organization », Spilka, R. (ed.), Writing in the Workplace. New Research Perspectives, Carbondale, Southern Illinois University Press, pp. 4155.

Maingueneau, D. (1991) : L'analyse de discours, Paris, Hachette Supérieur. - (2002) : "Communauté discursive ", Charaudeau, P. \& Maingueneau, D. (éds), Dictionnaire d'analyse du discours, Paris, Seuil, pp. 104-106.

Miller, C. \& ChARney, D. (2008) : «Persuasion, audience, and argument », Bazerman, C. (ed.), Handbook of Research on Writing. History, Society, School, Individual, Text, New York, Lawrence Erlbaum Associates, pp. 583-598.

Olive, T. \& PiOLAT, A. (2003) : «Activation des processus rédactionnels et qualité des textes ", Le langage et L'Homme 38/2, pp. 191-203. 
Perelman, C. et OlBRechts-TyteCA, L. ([1988], 1992, $5^{\mathrm{e}}$ édition) : Traité de l'argumentation, Bruxelles, Éditions de l'Université de Bruxelles.

Plane, D. (2006) : «Singularités et constants de la production d'écrit. L'écriture comme traitement de contraintes », Laffont-Terranova, J. \& Collin, D. (éds.) Didactique de l'écrit. La construction des savoirs et le sujet-écrivant, Namur, Presses universitaires de Namur, pp. 33-54.

Plantin, C. (1996) : L'argumentation, Mémo, Paris, Seuil.

POUIT, D. \& GOLDER, C. (1996) : "Peut-on faciliter l'argumentation écrite ? Effets d'un schéma de texte, d'une liste d'idées et d'un thème familier », Archives de Psychologie 64, pp. 179-199.

RoBRIEUX, J.-J. (2000, $2^{\mathrm{e}}$ édition) : Rhétorique et argumentation, Paris, Nathan Université.

SPILKA, R. (ed.). (1993) : Writing in the Workplace. New Research Perspectives. Carbondale, Southern Illinois University Press.

S TEIN, N., BERNAS, R., CALICCHIA, D. \& WRIGHT, A. (1996) : «Understanding and resolving arguments : The dynamics of negociation », Britton, B.K., Models of Understanding Text, Mahwah, NJ, Lawrence Erlbaum Associates, pp. 257-288.

STEIN, N.L. \& MiLler, C.A. (1993a) : «The development of memory and reasoning skill in argumentative contexts : Evaluating, explaining, and generating evidence ", Glaser, R. (Ed.), Advances in instructional psychology, vol.4, Hilldale, NJ, Lawrence Erlbaum Associates, pp. 285-335.

STEIN, N.L. \& MiLLER, C.A. (1993b) : «A theory of argumentative understanding : Relationships among position preference, judgments of goodness, memory and resoning », Argumentation, 7, pp. 183-204.

SWALES, J. (1996) : « Discourse community», Heilker, P. \& Vandenberg, P. (eds.), Keywords in Composition Studies, Portsmouth, NH, Boynton/Cook Publishers, pp. 67-70.

VignAUX, G. (1988) : Le discours acteur du monde. Énonciation, argumentation et cognition, Gap, Ophrys.

Voss, J. F., Vesonder, G. T., \& SpILICH, G. T. (1980) : « Text generation and recall by high knowledge and low knowledge individuals », Journal of Verbal Learning and Verbal Behavior 17, pp. 651-667. 